\title{
Large Sets of Hamilton Cycle and Path Decompositions of Complete Bipartite Graphs
}

\author{
Hongtao Zhao • Qingde Kang
}

Received: 27 April 2010 / Revised: 10 September 2011 / Published online: 5 October 2011

(C) The Author(s) 2011. This article is published with open access at Springerlink.com

\begin{abstract}
In this paper, we determine the existence spectrums for large sets of Hamilton cycle and path (resp. directed Hamilton cycle and path) decompositions of $\lambda K_{m, n}$ (resp. $\lambda K_{m, n}^{*}$ ).
\end{abstract}

Keywords Large set · Hamilton cycle $\cdot$ Hamilton path · Decomposition · Complete automorphism group

\section{Introduction}

Throughout this paper, let $\lambda K_{m, n}$ (resp. $\lambda K_{m, n}^{*}$ ) be the complete bipartite multigraph (resp. multi-digraph) with two partite sets $Z_{m}$ and $\bar{Z}_{n}$. Without loss of generality, we suppose $m \geq n$ in $\lambda K_{m, n}$ and $\lambda K_{m, n}^{*}$. In this paper, we use the convention that if $\lambda$ is not specified, then $\lambda=1$. A $k$-cycle (resp. $k$-path) is a subgraph of $K_{m, n}$ with $k$ vertices $x_{1}, x_{2}, \ldots, x_{k}$ and $k$ edges $\left\{x_{1}, x_{2}\right\}, \ldots,\left\{x_{k-1}, x_{k}\right\},\left\{x_{k}, x_{1}\right\}$ (resp. $k-1$ edges $\left\{x_{1}, x_{2}\right\}, \ldots,\left\{x_{k-1}, x_{k}\right\}$ ), which is denoted by $\left(x_{1}, x_{2}, \ldots, x_{k}\right)$ (resp. $\left[x_{1}, x_{2}, \ldots, x_{k}\right]$ ). A directed $k$-cycle (resp. directed $k$-path) is a subgraph of $K_{m, n}^{*}$

Research supported by NSFC Grant 10901051, NSFC Grant 10971051 and the Fundamental Research Funds for the Central Universities (No.10ML37).

H. Zhao $(\varangle)$

School of Mathematics and Physics, North China Electric Power University, Beijing 102206,

People's Republic of China

e-mail: ht_zhao@163.com

Q. Kang

Institute of Mathematics, Hebei Normal University, Shijiazhuang 050016,

People's Republic of China

e-mail: qdkang@heinfo.net 
with $k$ vertices $x_{1}, x_{2}, \ldots, x_{k}$ and $k$ arcs $\left(x_{1}, x_{2}\right),\left(x_{2}, x_{3}\right), \ldots,\left(x_{k-1}, x_{k}\right),\left(x_{k}, x_{1}\right)$ [resp. $\left.k-1 \operatorname{arcs}\left(x_{1}, x_{2}\right), \ldots,\left(x_{k-1}, x_{k}\right)\right]$, which is denoted by $\left\langle x_{1}, x_{2}, \ldots, x_{k}\right\rangle$ (resp. $\left.\prec x_{1}, x_{2}, \ldots, x_{k} \succ\right)$. When $k=m+n$, a (directed) $k$-cycle is called a (directed) Hamilton cycle, a (directed) $k$-path is called a (directed) Hamilton path. It is easy to see that

if there exists a Hamilton cycle (resp. directed Hamilton cycle) in $K_{m, n}$ (resp. $\left.K_{m, n}^{*}\right)$, then $m=n$;

if there exists a Hamilton path (resp. directed Hamilton path) in $K_{m, n}\left(\right.$ resp. $\left.K_{m, n}^{*}\right)$, then $m=n$ or $n+1$.

A Hamilton cycle (resp. directed Hamilton cycle) decomposition of $\lambda K_{n, n}$ (resp. $\left.\lambda K_{n, n}^{*}\right), \operatorname{HC}(n, n, \lambda)(\operatorname{resp} \operatorname{DHC}(n, n, \lambda))$, is a $\left(Z_{n} \cup \bar{Z}_{n}, \mathcal{A}\right)$, where $\mathcal{A}$ is a collection of Hamilton cycles (resp. directed Hamilton cycles), called blocks, in $K_{n, n}$ (resp. $\left.K_{n, n}^{*}\right)$, which form a partition of edge (resp. arc) set of $\lambda K_{n, n}\left(\operatorname{resp} . \lambda K_{n, n}^{*}\right)$. When $m=n$ or $n+1$, we can similarly define a Hamilton path (resp. directed Hamilton path) decomposition of $\lambda K_{m, n}$ (resp. $\left.\lambda K_{m, n}^{*}\right)$, which is denoted by $\operatorname{HP}(m, n, \lambda)$ (resp. $\operatorname{DHP}(m, n, \lambda))$. A decomposition is said to be simple if it contains no repeated blocks.

A large set of Hamilton cycle (resp. directed Hamilton cycle) decomposition of $\lambda K_{n, n}\left(\operatorname{resp} . \lambda K_{n, n}^{*}\right), \operatorname{LHC}(n, n, \lambda)[\operatorname{resp} \operatorname{LDHC}(n, n, \lambda)]$, is a partition of all Hamilton cycles (resp. directed Hamilton cycles) of $K_{n, n}$ (resp. $\left.K_{n, n}^{*}\right)$ into $\mathrm{HC}(n, n, \lambda) \mathrm{s}$ [resp. $\operatorname{DHC}(n, n, \lambda) \mathrm{s}]$. When $m=n$ or $n+1$, we can similarly define a large set of Hamilton path (resp. directed Hamilton path) decomposition of $\lambda K_{m, n}\left(\operatorname{resp} . \lambda K_{m, n}^{*}\right.$ ), which is denoted by $\operatorname{LHP}(m, n, \lambda)[\operatorname{resp} \operatorname{LDHP}(m, n, \lambda)]$. It is easy to see that every decomposition in a large set is simple.

Let $\lambda K_{n}$ (resp. $\lambda K_{n}^{*}$ ) denote the complete multigraph (resp. multi-digraph) on $n$ vertices. A Hamilton cycle (resp. path) of $K_{n}$ is a $n$-cycle (resp. $n$-path) of $K_{n}$. An almost Hamilton cycle (resp. path) of $K_{n}$ is a $(n-1)$-cycle [resp. $(n-1)$-path] of $K_{n}$. There are similar definitions of directed Hamilton cycle and path of $K_{n}^{*}$. As well, there are similar definitions of (almost) Hamilton cycle and path decomposition of $\lambda K_{n}$, of directed Hamilton cycle and path decomposition of $\lambda K_{n}^{*}$.

Lemma $1[1,10]$ There exists a large set of Hamilton cycle (resp. path) decompositions of $\lambda K_{n}\left(\lambda K_{n-1}\right)$ if and only if $2 \mid \lambda(n-1)$ and $\lambda \mid(n-2)$ !.

Lemma 2 [11] There exists a large set of almost Hamilton cycle decomposition of $2 K_{n}$ for any $n \equiv 0,1(\bmod 4)$ except $n=5$.

Lemma 3 [10] There exists a large set of directed Hamilton cycle (resp. path) decomposition of $\lambda K_{n}^{*}$ (resp. $\lambda K_{n-1}^{*}$ ) for any $n \geq 3$ and $n \neq 4,6$ with possible exceptions $n \in\{p+1:$ prime $p \geq 23\}$.

Lemma 4 [3] There exists an LHC(2m,2m,1) for any positive integer $m$.

There are many other classical problems about large sets. Please refer [6-8] for large sets of Steiner triple systems, [5] for large sets of Mendelsohn triple systems, [4] for large sets of transitive triple systems, etc. In this paper, we will determine the existence spectrums for large sets of Hamilton cycle and path (resp. directed Hamilton cycle and path) decompositions of $\lambda K_{m, n}\left(\operatorname{resp} . \lambda K_{m, n}^{*}\right)$. 


\section{Small Designs}

Obviously, an $\operatorname{HC}(n, n, \lambda)$ consists of $\frac{\lambda n^{2}}{2 n}=\frac{\lambda n}{2}$ blocks. Hence,

if there exists an $\operatorname{HC}(n, n, \lambda)$, then $\left\{\begin{array}{l}\operatorname{even} n \geq 2 \text { for any } \lambda \text {; } \\ \operatorname{odd} n \geq 3 \text { for even } \lambda\end{array}\right.$

So, the necessary conditions for the existence of a $\operatorname{DHC}(n, n, \lambda)$ are $n>1$ for any $\lambda$.

Lemma 5 There exists an $H C(2 m, 2 m, \lambda)$ for positive integers $m$ and $\lambda$.

Proof Define the collection $\mathcal{A}$ of the following $m$ Hamilton cycles

$$
C_{i}=(0, \overline{2 i}, 1, \overline{2 i+1}, \ldots, 2 m-1, \overline{2 i+2 m-1}), \quad 0 \leq i \leq m-1,
$$

where $\overline{2 i+j} \in \bar{Z}_{2 m}$ for $0 \leq i \leq m-1,0 \leq j \leq 2 m-1$. It is easy to verify that $\left(Z_{2 m} \cup \bar{Z}_{2 m}, \mathcal{A}\right)$ is an $\operatorname{HC}(2 m, 2 m, 1)$. Repeating every $C_{i} \lambda$ times, we obtain an $\mathrm{HC}(2 m, 2 m, \lambda)$.

Lemma 6 There exists an $H C(2 m+1,2 m+1,2 \lambda)$ for positive integers $m$ and $\lambda$.

Proof Define the collection $\mathcal{A}$ of the following $2 m+1$ Hamilton cycles

$$
D_{i}=(0, \bar{i}, 1, \overline{i+1}, \ldots, 2 m, \overline{i+2 m}), \quad 0 \leq i \leq 2 m,
$$

where $\overline{i+j} \in \bar{Z}_{2 m+1}$ for $0 \leq i, j \leq 2 m$. It is easy to verify that $\left(Z_{2 m+1} \cup \bar{Z}_{2 m+1}, \mathcal{A}\right)$ is an $\mathrm{HC}(2 m+1,2 m+1,2)$. Repeating every $D_{i} \lambda$ times, we obtain an $\operatorname{HC}(2 m+$ $1,2 m+1,2 \lambda)$.

Lemma 7 There exists a $\operatorname{DHC}(n, n, \lambda)$ for positive integers $n$ and $\lambda, n>1$.

Proof We use the structure in Lemma 1 of [9], define the collection $\mathcal{A}$ of the following $n$ directed Hamilton cycles

$$
C_{i}=\langle 0, \bar{i}, 1, \overline{i+1}, \ldots, n-1, \overline{i+n-1}\rangle, \quad 0 \leq i \leq n-1,
$$

where $\overline{i+j} \in \bar{Z}_{n}$ for $0 \leq i, j \leq n-1$. It is easy to verify that $\left(Z_{n} \cup \bar{Z}_{n}, \mathcal{A}\right)$ is a $\operatorname{DHC}(n, n, 1)$. Repeating every $C_{i} \lambda$ times, we obtain a $\operatorname{DHC}(n, n, \lambda)$.

It is clear that $|\mathcal{A}|=\frac{\lambda n(n-1)}{2 n-2}=\frac{\lambda n}{2}$ in an $\operatorname{HP}(n, n-1, \lambda)$. Hence,

$$
\text { if there exists an } \operatorname{HP}(n, n-1, \lambda) \text {, then }\left\{\begin{array}{l}
\operatorname{even} n \geq 2 \text { for any } \lambda \\
\operatorname{odd} n \geq 3 \text { for even } \lambda
\end{array}\right.
$$

Clearly, the necessary conditions for the existence of a $\operatorname{DHP}(n, n-1, \lambda)$ are $n>1$ for any $\lambda$. It is easy to see that the existence of an $\mathrm{HC}(n, n, \lambda)[\operatorname{resp} \operatorname{DHC}(n, n, \lambda)]$ is equivalent to the existence of an $\operatorname{HP}(n, n-1, \lambda)[\operatorname{resp} . \operatorname{DHP}(n, n-1, \lambda)]$. In Sects. 3 
and 4, we will show that the existence of an $\operatorname{LHC}(n, n, \lambda)[\operatorname{resp} \operatorname{LDHC}(n, n, \lambda)]$ is equivalent to the existence of $\operatorname{an} \operatorname{LHP}(n, n-1, \lambda)[\operatorname{resp} . \operatorname{LDHP}(n, n-1, \lambda)]$. So, the following lemma is an immediate consequence of Lemmas 5-7.

Lemma 8 There exist an $H P(2 m, 2 m-1, \lambda)$, an $H P(2 m+1,2 m, 2 \lambda)$ and a $\operatorname{DHP}(n, n-1, \lambda)$ for positive integers $m, n$ and $\lambda, n>1$.

$\operatorname{An} \operatorname{HP}(n, n, \lambda)$ consists of $\frac{\lambda n^{2}}{2 n-1}$ blocks. But, $\operatorname{gcd}\left(n^{2}, 2 n-1\right)=1$. Hence,

if there exists an $\operatorname{HP}(n, n, \lambda)$, then $(2 n-1) \mid \lambda$.

Similarly, the necessary condition for the existence of $\operatorname{DHP}(n, n, \lambda)$ is also $(2 n-1) \mid \lambda$.

Lemma 9 There exists an $\operatorname{HP}(n, n, \lambda(2 n-1))$ for positive integers $n$ and $\lambda$.

Proof Define the collection $\mathcal{A}$ of the following $n^{2}$ Hamilton paths

$$
C_{i, j}=[i, \bar{j}, i+1, \overline{j+1}, \ldots, i+n-1, \overline{j+n-1}], \quad 0 \leq i, j \leq n-1,
$$

where $i+k \in Z_{n}, \overline{j+k} \in \bar{Z}_{n}$ for $0 \leq i, j, k \leq n-1$. It is easy to verify that $\left(Z_{n} \cup \bar{Z}_{n}, \mathcal{A}\right)$ is an $\operatorname{HP}(n, n, 2 n-1)$. Repeating every $C_{i, j} \lambda$ times, we obtain an $\operatorname{HP}(n, n, \lambda(2 n-1))$.

Lemma 10 There exists a $\operatorname{DH} P(n, n, \lambda(2 n-1))$ for positive integers $n$ and $\lambda$.

Proof It is easy to see that the existence of an $\operatorname{HP}(n, n, \lambda(2 n-1))$ implies the existence of an $\operatorname{DHP}(n, n, \lambda(2 n-1))$.

In Lemmas 5-10, when $\lambda>1$, all decompositions are not simple (i.e., containing repeated blocks). In the following sections, we will mention the simple cases.

\section{$3 \operatorname{LHC}(n, n, \lambda)$ and $\operatorname{LHP}(n, n-1, \lambda)$}

Let $\operatorname{Sym}(S)$ be the symmetric group on a given set $S$. For a subgroup $T$ of $\operatorname{Sym}(S)$, the set of representatives of the right cosets for $T$ in $\operatorname{Sym}(S)$ is denoted by $\operatorname{Sym}_{T}(S)$. For any $s \in S$ and two permutations $\xi_{1}, \xi_{2} \in \operatorname{Sym}(S)$, define $\xi_{1} \xi_{2}(s)=\xi_{2}\left(\xi_{1}(s)\right)$.

Let $C=\left(x_{0}, \bar{x}_{0}, x_{1}, \bar{x}_{1}, \ldots, x_{n-1}, \bar{x}_{n-1}\right)$ be a Hamilton cycle of $K_{n, n}$, where $x_{i} \in Z_{n}, \bar{x}_{i} \in \bar{Z}_{n}$ for $0 \leq i \leq n-1$. For permutations $\xi \in \operatorname{Sym}\left(Z_{n}\right)$ and $\eta \in \operatorname{Sym}\left(\bar{Z}_{n}\right)$, denote $\xi C=\left(\xi\left(x_{0}\right), \bar{x}_{0}, \xi\left(x_{1}\right), \bar{x}_{1}, \ldots, \xi\left(x_{n-1}\right), \bar{x}_{n-1}\right)$ and $\eta C=\left(x_{0}, \eta\left(\bar{x}_{0}\right), x_{1}, \eta\left(\bar{x}_{1}\right), \ldots, x_{n-1}, \eta\left(\bar{x}_{n-1}\right)\right)$, respectively. Take

$$
\sigma=(1, n-1)(2, n-2) \cdots\left(\left\lfloor\frac{n-1}{2}\right\rfloor, n-\left\lfloor\frac{n-1}{2}\right\rfloor\right) \in \operatorname{Sym}\left(Z_{n}\right)
$$

which generates a subgroup $G=\langle\sigma\rangle$ of $\operatorname{Sym}\left(Z_{n}^{\prime}\right)$ with order two, where $Z_{n}^{\prime}=Z_{n} \backslash\{0\}$. Then, $\left|\operatorname{Sym}_{G}\left(Z_{n}^{\prime}\right)\right|=\frac{(n-1) !}{2}$. Let $\operatorname{Sym}_{G}\left(Z_{n}^{\prime}\right)=\left\{\sigma_{1}, \sigma_{2}, \ldots, \sigma_{(n-1) ! / 2}\right\}$. Below, by the 
shift-equivalence of Hamilton cycles, each Hamilton cycle in $K_{n, n}$ will be denoted by a fixed form as follows.

Under the action of $\operatorname{Sym}\left(\bar{Z}_{n}\right)$, all Hamilton cycles in $K_{n, n}$ can be separated into the following $\frac{(n-1) !}{2}$ orbits, where $\sigma_{i} \in \operatorname{Sym} m_{G}\left(Z_{n}^{\prime}\right)$.

$\mathcal{O}_{i}=\left\{\left(0, \eta(\overline{0}), \sigma_{i}(1), \eta(\overline{1}), \sigma_{i}(2), \eta(\overline{2}), \ldots, \sigma_{i}(n-1), \eta(\overline{n-1})\right): \eta \in \operatorname{Sym}\left(\bar{Z}_{n}\right)\right\}$.

Obviously, $\left|\mathcal{O}_{i}\right|=n$ ! for $1 \leq i \leq \frac{(n-1) !}{2}$. So, $\left|\operatorname{Sym}_{G}\left(Z_{n}^{\prime}\right)\right| \cdot\left|\mathcal{O}_{i}\right|=\frac{(n-1) ! n !}{2}$ is just the total number of distinct Hamilton cycles in $K_{n, n}$.

Let $\mathcal{A}$ be a collection of Hamilton cycles (resp. directed Hamilton cycles) in $K_{n, n}$ (resp. $\left.K_{n, n}^{*}\right)$. A subgroup $H$ of $\operatorname{Sym}\left(\bar{Z}_{n}\right)$ is called a complete automorphism group over $\bar{Z}_{n}$ of $\mathcal{A}$ if the following conditions are satisfied:

1. $\eta C \in \mathcal{A}$ for any $\eta \in H$ and $C \in \mathcal{A}$;

2. $\forall C, C^{\prime} \in \mathcal{B}$, if there exists $\eta \in \operatorname{Sym}\left(\bar{Z}_{n}\right)$ such that $\eta C=C^{\prime}$, then $\eta \in H$.

When $\mathcal{A}$ is a collection of Hamilton paths (resp. directed Hamilton paths) in $K_{n, n}$ (resp. $K_{n, n}^{*}$ ), we can similarly define the complete automorphism group for $\mathcal{A}$.

In the following discussions, $\mathcal{A}$ consists of all Hamilton cycles in some $\operatorname{HC}(n, n, \lambda)$. We now give a very useful lemma in this paper. The idea of the construction, introduced in [2], is to make use of symmetric groups.

Lemma 11 (1) If $\left(Z_{n} \cup \bar{Z}_{n}, \mathcal{A}\right)$ is an $H C(n, n, \lambda)$ then so is $\left(Z_{n} \cup \bar{Z}_{n}, \eta \mathcal{A}\right)$ (resp. $\left.\left(Z_{n} \cup \bar{Z}_{n}, \xi \mathcal{A}\right)\right)$, where $\eta \in \operatorname{Sym}\left(\bar{Z}_{n}\right), \eta \mathcal{A}=\{\eta C: C \in \mathcal{A}\}($ resp. $\xi \in$ $\left.\operatorname{Sym}\left(Z_{n}\right), \xi \mathcal{A}=\{\xi C: C \in \mathcal{A}\}\right)$;

(2) If the system $\mathcal{A}$ is simple and has a complete automorphism group $H$ over $\bar{Z}_{n}$, then all Hamilton cycles in $\left\{\eta \mathcal{A}: \eta \in \operatorname{Sym}_{H}\left(\bar{Z}_{n}\right)\right\}$ are pairwise distinct.

Proof (1) The permutation $\eta$ on $\bar{Z}_{n}$ induces a permutation on the set $\left(\bar{Z}_{n} \times\right.$ $\left.\bar{Z}_{n}\right) \backslash\left\{(y, y): y \in \bar{Z}_{n}\right\}$. Hence, the system $\left(Z_{n} \cup \bar{Z}_{n}, \eta \mathcal{A}\right)$ is also an $\operatorname{HC}(n, n, \lambda)$ by the definition. For $\xi \in \operatorname{Sym}\left(Z_{n}\right)$, the proof is similar.

(2) Suppose there exist $C, C^{\prime} \in \mathcal{A}$ and $\eta_{1} \neq \eta_{2} \in \operatorname{Sym}_{H}\left(\bar{Z}_{n}\right)$ such that $\eta_{1} C=$ $\eta_{2} C^{\prime}$. Then $\left(\eta_{1} \eta_{2}^{-1}\right) C=C^{\prime}$ and $\eta_{1} \eta_{2}^{-1} \in H$ by the definition of complete automorphism group $H$ over $\bar{Z}_{n}$. This implies $H \eta_{1}=H \eta_{2}$, i.e., $\eta_{1}$ and $\eta_{2}$ belong to the same coset, which is a contradiction.

An $\mathrm{HC}(n, n, \lambda)$ contains $\frac{\lambda n}{2}$ Hamilton cycles. The total number of distinct Hamilton cycles in $K_{n, n}$ is $\frac{(n-1) ! n !}{2}$. Hence, an $\operatorname{LHC}(n, n, \lambda)$ contains $((n-1) !)^{2} / \lambda$ pairwise disjoint $\mathrm{HC}(n, n, \lambda)$ s. Clearly, there exists an $\operatorname{LHC}(n, n, \lambda)$ only if

$$
\lambda \mid((n-1) !)^{2} \text { and }\left\{\begin{array}{l}
\text { even } n \geq 2 \text { for any } \lambda \\
\text { odd } n \geq 3 \text { for even } \lambda .
\end{array}\right.
$$

The conditions are also necessary for the existence of $\operatorname{LHP}(n, n-1, \lambda)$. Thus, the existence spectrum for $\operatorname{LHC}(n, n, \lambda)[\operatorname{resp} \operatorname{LHP}(n, n-1, \lambda)]$ only depends on two cases: even $n \geq 2$ for $\lambda=1$ and odd $n \geq 3$ for $\lambda=2$.

Lemma 12 There exists an $\operatorname{LHC}(2 m+1,2 m+1,2)$ for any positive integer $m$. 
Proof Take the $\mathrm{HC}(2 m+1,2 m+1,2)=\left(Z_{2 m+1} \cup \bar{Z}_{2 m+1}, \mathcal{A}\right)$ constructed in Lemma 6 as the base small set, where $\mathcal{A}=\left\{D_{0}, D_{1}, \ldots, D_{2 m}\right\}$. Let $\tau=(\overline{0}, \overline{1}, \ldots, \overline{2 m}) \in$ $\operatorname{Sym}\left(\bar{Z}_{2 m+1}\right)$, which generates a subgroup $H=\langle\tau\rangle$ of $\operatorname{Sym}\left(\bar{Z}_{2 m+1}\right)$ with order $2 m+$ 1. Clearly, $D_{j}=\tau^{j-i} D_{i}$ for $i, j \in Z_{2 m+1}$. Now, we have shown that $H$ is a complete automorphism group of $\mathcal{A}$ over $\bar{Z}_{2 m+1}$. Let $\operatorname{Sym}_{H}\left(\bar{Z}_{2 m+1}\right)=\left\{\tau_{1}, \tau_{2}, \ldots, \tau_{(2 m) !}\right\}$, where $\tau_{1}$ is identical permutation. Let $\operatorname{Sym}_{G}\left(Z_{2 m+1}^{\prime}\right)=\left\{\sigma_{1}, \sigma_{2}, \ldots, \sigma_{(2 m) ! / 2}\right\}$ (refer the beginning of this section).

Define

$$
\Omega_{i, j}=\left\{\sigma_{i} \tau_{j} D_{0}, \sigma_{i} \tau_{j} D_{1}, \ldots, \sigma_{i} \tau_{j} D_{2 m}\right\}, \quad 1 \leq i \leq \frac{(2 m) !}{2}, \quad 1 \leq j \leq(2 m) ! .
$$

Each $\Omega_{i, j}$ is an $\mathrm{HC}(2 m+1,2 m+1,2)$ by Lemma 11 (1). Similarly, we can prove that $H$ is a complete automorphism group of $\Omega_{i, 1}$, over $\bar{Z}_{2 m+1}$, for $1 \leq i \leq \frac{(2 m) !}{2}$. We have the facts:

* all Hamilton cycles in each $\Omega_{i, j}$ fall into orbit $\mathcal{O}_{i}$, where $1 \leq i \leq \frac{(2 m) !}{2}, 1 \leq j \leq$ $(2 m)$ !;

* for given $\sigma_{i}$, all Hamilton cycles in $\left\{\Omega_{i, j}: 1 \leq j \leq(2 m) !\right\}$ are distinct by Lemma $11(2)$.

As well, $\left|\operatorname{Sym}_{G}\left(Z_{2 m+1}^{\prime}\right)\right| \cdot\left|\operatorname{Sym}_{H}\left(\bar{Z}_{2 m+1}\right)\right|=\left|\bigcup_{i, j} \Omega_{i, j}\right|=\frac{((2 m) !)^{2}}{2}$, which is just the desired number of disjoint $\mathrm{HC}(2 m+1,2 m+1,2) \mathrm{s}$ in an $\mathrm{LHC}(2 m+1,2 m+1,2)$. Therefore, by these facts, an $\operatorname{LHC}(2 m+1,2 m+1,2)$ is constructed.

Theorem 1 There exists an $\operatorname{LHC}(n, n, \lambda)$ if and only if $\lambda \mid((n-1) !)^{2}$ and

$$
\left\{\begin{array}{l}
\text { even } n \geq 2 \text { for any } \lambda \\
\text { odd } n \geq 3 \text { for even } \lambda
\end{array} .\right.
$$

Proof The necessity has been shown before Lemma 12, the sufficiency is proved below.

For even $n \geq 2$, there exists an $\operatorname{LHC}(n, n, 1)=\left\{\left(Z_{n} \cup \bar{Z}_{n}, \mathcal{A}_{i}\right): 1 \leq i \leq\right.$ $\left.((n-1) !)^{2}\right\}$ by Lemma 4 . Define

$$
\mathcal{B}_{k}=\bigcup_{i=k \lambda+1}^{(k+1) \lambda} \mathcal{A}_{i}, 0 \leq k \leq((n-1) !)^{2} / \lambda-1 \text {, }
$$

then $\left\{\left(Z_{n} \cup \bar{Z}_{n}, \mathcal{B}_{k}\right): 0 \leq k \leq((n-1) !)^{2} / \lambda-1\right\}$ is an $\operatorname{LHC}(n, n, \lambda)$, where $\lambda \mid((n-$ $1) !)^{2}$.

For odd $n \geq 3$ and even $\lambda \mid((n-1) !)^{2}$, there exists an $\operatorname{LHC}(n, n, 2)$ $=\left\{\left(Z_{n} \cup \bar{Z}_{n}, \mathcal{A}_{i}\right): 1 \leq i \leq \frac{((n-1) !)^{2}}{2}\right\}$ by Lemma 12. Define

$$
\mathcal{B}_{k}=\bigcup_{i=\frac{k \lambda}{2}+1}^{(k+1) \frac{\lambda}{2}} \mathcal{A}_{i}, \quad 0 \leq k \leq((n-1) !)^{2} / \lambda-1
$$


then $\left\{\left(Z_{n} \cup \bar{Z}_{n}, \mathcal{B}_{k}\right): 0 \leq k \leq((n-1) !)^{2} / \lambda-1\right\}$ is an $\operatorname{LHC}(n, n, \lambda)$. This completes the proof.

Theorem 2 There exists an $\operatorname{LH} P(n, n-1, \lambda)$ if and only if $\lambda \mid((n-1) !)^{2}$ and

$$
\left\{\begin{array}{l}
\text { even } n \geq 2 \text { for any } \lambda \\
\text { odd } n \geq 3 \text { for even } \lambda
\end{array} .\right.
$$

Proof We start proving the sufficiency first. By Theorem 1, there exists an $\operatorname{LHC}(n, n, \lambda)=\left\{\left(Z_{n} \cup \bar{Z}_{n}, \mathcal{A}_{i}\right): 1 \leq i \leq((n-1) !)^{2} / \lambda\right\}$. Delete the element $\overline{0}$ from the set $\bar{Z}_{n}$, let $\bar{Z}_{n}^{\prime}=\bar{Z}_{n} \backslash\{\overline{0}\}$. Then, each Hamilton cycle in each $\mathcal{A}_{i}$ will become a Hamilton path of $K_{n, n-1}$ with two partite sets $Z_{n}, \bar{Z}_{n}^{\prime}$, and each Hamilton cycle decomposition $\left(Z_{n} \cup \bar{Z}_{n}, \mathcal{A}_{i}\right)$ of $\lambda K_{n, n}$ will become a Hamilton path decomposition $\left(Z_{n} \cup \bar{Z}_{n}^{\prime}, \mathcal{A}_{i}^{\prime}\right)$ of $\lambda K_{n, n-1}$. It is easy to verify that $\left\{\left(Z_{n} \cup \bar{Z}_{n}^{\prime}, \mathcal{A}_{i}^{\prime}\right): 1 \leq i \leq\right.$ $\left.((n-1) !)^{2} / \lambda\right\}$ indeed forms an $\operatorname{LHP}(n, n-1, \lambda)$. As for the necessity, see before Lemma 12. The conclusion holds.

Corollary 1 (1) There exist simple $H C(2 m, 2 m, \lambda)$ and simple $H P(2 m, 2 m-1, \lambda)$ if and only if $1 \leq \lambda \leq((2 m-1) !)^{2}$;

(2) There exist simple $H C(2 m+1,2 m+1,2 \lambda)$ and simple $H P(2 m+1,2 m, 2 \lambda)$ if and only if $1 \leq \lambda \leq((2 m) !)^{2} / 2$.

\section{$4 \operatorname{LDHC}(n, n, \lambda)$ and $\operatorname{LDHP}(n, n-1, \lambda)$}

For $\xi \in \operatorname{Sym}\left(Z_{n}\right), \eta \in \operatorname{Sym}\left(\bar{Z}_{n}\right)$ and a directed Hamilton cycle $C=\left\langle x_{0}, \bar{x}_{0}, \ldots\right.$, $\left.x_{n-1}, \bar{x}_{n-1}\right\rangle$ of $K_{n, n}^{*}$, where $x_{i} \in Z_{n}, \bar{x}_{i} \in \bar{Z}_{n}$ for $0 \leq i \leq n-1$, the definitions of $\xi C$ and $\eta C$ are similar to those introduced in Sect. 3. Let $\bar{Z}_{n}^{\prime}=Z_{n} \backslash\{0\}$. Then, by the shift-equivalence of directed Hamilton cycles, each directed Hamilton cycle in $K_{n, n}^{*}$ will be denoted by a fixed form as follows.

Under the action of $\operatorname{Sym}\left(\bar{Z}_{n}\right)$, all directed Hamilton cycles in $K_{n, n}^{*}$ can be separated into the following orbits:

$\mathcal{O}_{i}^{\prime}=\left\{\left\langle 0, \eta(\overline{0}), \sigma_{i}(1), \eta(\overline{1}), \ldots, \sigma_{i}(n-1), \eta(\overline{n-1})\right\rangle: \eta \in \operatorname{Sym}\left(\bar{Z}_{n}\right)\right\}, \sigma_{i} \in \operatorname{Sym}\left(Z_{n}^{\prime}\right)$.

It is easy to see that $\left|\mathcal{O}_{i}^{\prime}\right|=n$ ! for any $\sigma_{i} \in \operatorname{Sym}\left(Z_{n}^{\prime}\right)$. And, $\left|\operatorname{Sym}\left(Z_{n}^{\prime}\right)\right| \cdot\left|\mathcal{O}_{i}^{\prime}\right|=$ $(n-1) ! n$ ! is just the total number of distinct directed Hamilton cycles in $K_{n, n}^{*}$.

Similarly to Lemma 11, we can prove the following one which is on oriented cycles.

Lemma 13 (1) If $\left(Z_{n} \cup \bar{Z}_{n}, \mathcal{A}\right)$ is a $\operatorname{DHC}(n, n, \lambda)$ then so is $\left(Z_{n} \cup \bar{Z}_{n}, \eta \mathcal{A}\right)[$ resp. $\left.\left(Z_{n} \cup \bar{Z}_{n}, \xi \mathcal{A}\right)\right]$, where $\eta \in \operatorname{Sym}\left(\bar{Z}_{n}\right)\left[\operatorname{resp} . \xi \in \operatorname{Sym}\left(Z_{n}\right)\right]$;

(2) If the system $\mathcal{A}$ is simple and it has a complete automorphism group $H$ over $\bar{Z}_{n}$, then all directed Hamilton cycles in $\left\{\eta \mathcal{A}: \eta \in \operatorname{Sym}_{H}\left(\bar{Z}_{n}\right)\right\}$ are pairwise distinct.

A $\operatorname{DHC}(n, n, \lambda)$ contains $\lambda n$ directed Hamilton cycles. The total number of distinct directed Hamilton cycles in $K_{n, n}^{*}$ is $(n-1) ! n !$. Hence, an $\operatorname{LDHC}(n, n, \lambda)$ 
contains $((n-1) !)^{2} / \lambda$ pairwise disjoint $\operatorname{DHC}(n, n, \lambda)$ s. Clearly, there exists an $\operatorname{LDHC}(n, n, \lambda)$ only if $\lambda \mid((n-1) !)^{2}$. The conditions are also necessary for the existence of $\operatorname{LDHP}(n, n-1, \lambda)$. Therefore, the existence spectrum for $\operatorname{LDHC}(n, n, \lambda)$ and $\operatorname{LDHP}(n, n-1, \lambda)$ only depends on one case: $\lambda=1$ and $n \geq 1$.

Lemma 14 There exists an $\operatorname{LDHC}(n, n, 1)$ for any positive integer $n$.

Proof Take the $\operatorname{DHC}(n, n, 1)=\left(Z_{n} \cup \bar{Z}_{n}, \mathcal{A}\right)$ constructed in Lemma 7 as the base small set, where $\mathcal{A}=\left\{C_{0}, C_{1}, \ldots, C_{n-1}\right\}$. Let $\tau=(\overline{0}, \overline{1}, \ldots, \overline{n-1}) \in \operatorname{Sym}\left(\bar{Z}_{n}\right)$, which generates a subgroup $H=\langle\tau\rangle$ of $\operatorname{Sym}\left(\bar{Z}_{n}\right)$ with order $n$. Clearly, $C_{j}=\tau^{j-i} C_{i}$ for $i, j \in Z_{n}$. Now, we have shown that $H$ is a complete automorphism group of $\mathcal{A}$ over $\bar{Z}_{n}$. Let $\operatorname{Sym}_{H}\left(\bar{Z}_{n}\right)=\left\{\tau_{1}, \tau_{2}, \ldots, \tau_{(n-1) !}\right\}$, where $\tau_{1}$ is identical permutation. Let $\operatorname{Sym}\left(Z_{n}^{\prime}\right)=\left\{\sigma_{1}, \sigma_{2}, \ldots, \sigma_{(n-1) !}\right\}$. Define

$$
\Omega_{i, j}=\left\{\sigma_{i} \tau_{j} C_{0}, \sigma_{i} \tau_{j} C_{1}, \ldots, \sigma_{i} \tau_{j} C_{n-1}\right\}, \quad 1 \leq i, j \leq(n-1) !
$$

Each $\Omega_{i, j}$ is a $\operatorname{DHC}(n, n, 1)$ by Lemma $13(1)$. Similarly, we can prove that $H$ is a complete automorphism group of $\Omega_{i, 1}$ over $\bar{Z}_{n}$ for $1 \leq i \leq(n-1)$ !. We have the following facts:

* all directed Hamilton cycles in each $\Omega_{i, j}$ fall into orbit $\mathcal{O}_{i}^{\prime}$, where $1 \leq i, j \leq$ $(n-1)$ !;

* all directed Hamilton cycles in $\left\{\Omega_{i, j}: 1 \leq j \leq(n-1)\right.$ ! $\}$ are distinct by Lemma $13(2)$.

As well, $\left|\operatorname{Sym}\left(Z_{n}^{\prime}\right)\right| \cdot\left|\operatorname{Sym}_{H}\left(\bar{Z}_{n}\right)\right|=\left|\bigcup_{i, j} \Omega_{i, j}\right|=((n-1) !)^{2}$, which is just the number of disjoint $\operatorname{DHC}(n, n, 1) \mathrm{s}$ in an $\operatorname{LDHC}(n, n, 1)$. Therefore, an $\operatorname{LDHC}(n, n, 1)$ is constructed.

Similar to Theorems 1, 2 and Corollary 1, we can obtain the following conclusion. The proof is similar.

Theorem 3 There exists an $\operatorname{LDHC}(n, n, \lambda)$ if and only if $\lambda \mid((n-1) !)^{2}$.

Theorem 4 There exists an $\operatorname{LDHP}(n, n-1, \lambda)$ if and only if $\lambda \mid((n-1) !)^{2}$.

Corollary 2 There exist simple $\operatorname{DHC}(n, n, \lambda)$ and simple $\operatorname{DHP}(n, n-1, \lambda)$ if and only if $1 \leq \lambda \leq((n-1) !)^{2}$.

\section{$5 \operatorname{LHP}(n, n, \lambda)$ and $\operatorname{LDHP}(n, n, \lambda)$}

For $\xi \in \operatorname{Sym}\left(Z_{n}\right), \eta \in \operatorname{Sym}\left(\bar{Z}_{n}\right)$ and a Hamilton path $C=\left[x_{0}, \bar{x}_{0}, \ldots, x_{n-1}, \bar{x}_{n-1}\right]$ of $K_{n, n}$, where $x_{i} \in Z_{n}, \bar{x}_{i} \in \bar{Z}_{n}$ for $0 \leq i \leq n-1$, the definitions of $\xi C$ and $\eta C$ are similar to those introduced in Sect. 3. Take $\sigma=(0,1, \ldots, n-1) \in \operatorname{Sym}\left(Z_{n}\right)$, which generates a subgroup $G=\langle\sigma\rangle$ of $\operatorname{Sym}\left(Z_{n}\right)$ with order $n$. Then, $\left|\operatorname{Sym} m_{G}\left(Z_{n}\right)\right|=$ $(n-1)$ ! and $\operatorname{Sym}\left(Z_{n}\right)$ can be partitioned into $(n-1)$ ! right cosets: $\operatorname{Sym}\left(Z_{n}\right)=$ $\bigcup_{i=1}^{(n-1) !} G_{i}$, where $G_{i}=\left\{\sigma_{i, 0}, \sigma_{i, 1}, \ldots, \sigma_{i, n-1}\right\}, 1 \leq i \leq(n-1)$ !. We can modify the sequence $\sigma_{i, 0}, \sigma_{i, 1}, \ldots, \sigma_{i, n-1}$ such that $\sigma_{i, j+1}=\sigma \sigma_{i, j}$ for $j \in Z_{n}$. Furthermore, 
$\sigma_{i, j}=\sigma^{j-k} \sigma_{i, k}$ for $j, k \in Z_{n}$. Let $\operatorname{Sym}_{G}\left(Z_{n}\right)=\left\{\sigma_{1,0}, \sigma_{2,0}, \ldots, \sigma_{(n-1) !, 0}\right\}$, where $\sigma_{1,0}$ is identical permutation. Below, by the shift-equivalence of Hamilton paths, each Hamilton path in $K_{n, n}$ will be denoted by a fixed form as follows.

Under the action of $\operatorname{Sym}\left(\bar{Z}_{n}\right)$, all Hamilton paths in $K_{n, n}$ can be separated into the following orbit families:

$$
\overline{\mathcal{O}}_{i}=\left\{\mathcal{O}_{i, j}: 0 \leq j \leq n-1\right\}, \quad 1 \leq i \leq(n-1) !, \text { where }
$$

$\mathcal{O}_{i, j}=\left\{\left[\sigma_{i, j}(0), \eta(\overline{0}), \sigma_{i, j}(1), \eta(\overline{1}), \ldots, \sigma_{i, j}(n-1), \eta(\overline{n-1})\right]: \eta \in \operatorname{Sym}\left(\bar{Z}_{n}\right)\right\}$. It is easy to see that $\left|\overline{\mathcal{O}}_{i}\right|=n$ and $\left|\mathcal{O}_{i, j}\right|=n$ ! for $1 \leq i \leq(n-1)$ !, $0 \leq j \leq n-1$. The number of right cosets is $(n-1)$ !. Then, $(n-1) ! \cdot\left|\overline{\mathcal{O}}_{i}\right| \cdot\left|\mathcal{O}_{i, j}\right|=(n !)^{2}$ is just the total number of distinct Hamilton paths in $K_{n, n}$.

The next lemma is an analog of Lemma 11 too. Its proof is similar.

Lemma 15 (1) If $\left(Z_{n} \cup \bar{Z}_{n}, \mathcal{A}\right)$ is an $H P(n, n, \lambda)$ then so is $\left(Z_{n} \cup \bar{Z}_{n}, \eta \mathcal{A}\right)$ [resp. $\left.\left(Z_{n} \cup \bar{Z}_{n}, \xi \mathcal{A}\right)\right]$, where $\eta \in \operatorname{Sym}\left(\bar{Z}_{n}\right)\left[\operatorname{resp} . \xi \in \operatorname{Sym}\left(Z_{n}\right)\right]$;

(2) If the system $\mathcal{A}$ is simple and it has a complete automorphism group $H$ over $\bar{Z}_{n}$, then all Hamilton paths in $\left\{\eta \mathcal{A}: \eta \in \operatorname{Sym}_{H}\left(\bar{Z}_{n}\right)\right\}$ are pairwise distinct.

$\operatorname{An} \operatorname{HP}(n, n, \lambda)$ contains $\frac{\lambda n^{2}}{2 n-1}$ Hamilton paths. The total number of distinct Hamilton paths in $K_{n, n}$ is $(n !)^{2}$. Hence, an $\operatorname{LHP}(n, n, \lambda)$ contains $(2 n-1)((n-1) !)^{2} / \lambda$ pairwise disjoint $\operatorname{HP}(n, n, \lambda) \mathrm{s}$. Clearly, there exists an $\operatorname{LHP}(n, n, \lambda)$ only if $\lambda \mid(2 n-$ $1)((n-1) !)^{2}$ and $(2 n-1) \mid \lambda$. The conditions are also necessary for the existence of $\operatorname{LDHP}(n, n, \lambda)$. Therefore, the existence spectrum for $\operatorname{LHP}(n, n, \lambda)$ and $\operatorname{LDHP}(n, n, \lambda)$ only depends on one case: $\lambda=2 n-1$ and $n \geq 1$.

Lemma 16 There exists an $L H P(n, n, 2 n-1)$ for any positive integer $n$.

Proof Take the $\operatorname{HP}(n, n, 2 n-1)=\left(Z_{n} \cup \bar{Z}_{n}, \mathcal{A}\right)$ constructed in Lemma 9 as the base small set, where $\mathcal{A}=\left\{C_{i, j}: 0 \leq i, j \leq n-1\right\}$. Let $\tau=(\overline{0}, \overline{1}, \ldots, \overline{n-1}) \in$ $\operatorname{Sym}\left(\bar{Z}_{n}\right)$, which generates a subgroup $H=\langle\tau\rangle$ of $\operatorname{Sym}\left(\bar{Z}_{n}\right)$ with order $n$. Clearly, $C_{i, j}=\tau^{j-k} C_{i, k}$ for $i, j, k \in Z_{n}$. Now, we have shown that $H$ is a complete automorphism group over $\bar{Z}_{n}$ of $\mathcal{A}$. Let $\operatorname{Sym}_{H}\left(\bar{Z}_{n}\right)=\left\{\tau_{1}, \tau_{2}, \ldots, \tau_{(n-1) !}\right\}$, where $\tau_{1}$ is identical permutation. As well, let $\operatorname{Sym} m_{G}\left(Z_{n}\right)=\left\{\sigma_{1,0}, \sigma_{2,0}, \ldots, \sigma_{(n-1) !, 0}\right\}$ (refer the beginning of this section), where $\sigma_{1,0}$ is identical permutation too. Define

$$
\Omega_{i, j}=\left\{\sigma_{i, 0} \tau_{j} C_{k, l}: 0 \leq k, l \leq n-1\right\}, \quad 1 \leq i, j \leq(n-1) !
$$

Each $\Omega_{i, j}$ is an $\operatorname{HP}(n, n, 2 n-1)$ by Lemma $15(1)$. Similarly, we can prove that $H$ is a complete automorphism group of $\Omega_{i, 1}$ over $\bar{Z}_{n}$ for $1 \leq i \leq(n-1)$ !. We have the following facts.

* For given $\sigma_{i, 0}, 1 \leq i \leq(n-1)$ !, all Hamilton paths in $\Omega_{i, j}$ fall into orbit family $\overline{\mathcal{O}}_{i}$, where $1 \leq j \leq(n-1)$ !. In fact, in $\Omega_{i, j}$, for given $l \in Z_{n}$,

$$
\begin{aligned}
\sigma_{i, 0} \tau_{j} C_{k+1, l} & =\sigma_{i, 0} \tau_{j}\left(\sigma C_{k, l}\right)=\sigma \sigma_{i, 0} \tau_{j} C_{k, l}=\sigma_{i, 1} \tau_{j} C_{k, l} \text { for } k \in Z_{n}, \\
\sigma_{i, 0} \tau_{j} C_{k_{2}, l} & =\sigma_{i, 0} \tau_{j}\left(\sigma^{k_{2}-k_{1}} C_{k_{1}, l}\right)=\sigma^{k_{2}-k_{1}} \sigma_{i, 0} \tau_{j} C_{k_{1}, l} \\
& =\sigma_{i, k_{2}-k_{1}} \tau_{j} C_{k_{1}, l} \text { for } k_{1}, k_{2} \in Z_{n}
\end{aligned}
$$


That is to say, the $n$ Hamilton paths $\sigma_{i, 0} \tau_{j} C_{k, 0}, \sigma_{i, 0} \tau_{j} C_{k, 1}, \ldots, \sigma_{i, 0} \tau_{j} C_{k, n-1}$ belong to orbit $\mathcal{O}_{i, k}$, which is a member of orbit family $\overline{\mathcal{O}}_{i}$.

* For given $\sigma_{i, 0}, 1 \leq i \leq(n-1)$ !, all Hamilton paths in $\left\{\Omega_{i, j}: 1 \leq j \leq(n-1) !\right\}$ are pairwise distinct by Lemma 15 (2).

As well, $\left|\operatorname{Sym}_{G}\left(Z_{n}\right)\right| \cdot\left|\operatorname{Sym}_{H}\left(\bar{Z}_{n}\right)\right|=\left|\bigcup_{i, j} \Omega_{i, j}\right|=((n-1) !)^{2}$, which is just the desired number of disjoint $\operatorname{HP}(n, n, 2 n-1) \mathrm{s}$ in an $\operatorname{LHP}(n, n, 2 n-1)$. Therefore, by the facts, an $\operatorname{LHP}(n, n, 2 n-1)$ is constructed.

Theorem 5 There exists an $\operatorname{LHP}(n, n, \lambda)$ if and only if $\lambda \mid(2 n-1)((n-1) !)^{2}$ and $(2 n-1) \mid \lambda$.

Proof Combining Lemma 16 and the necessity for the existence of $\operatorname{LHP}(n, n, \lambda)$, we obtain the conclusion. The proof is similar to that of Theorem 1.

Theorem 6 There exists an $\operatorname{LDHP}(n, n, \lambda)$ if and only if $\lambda \mid(2 n-1)((n-1) !)^{2}$ and $(2 n-1) \mid \lambda$.

Proof If $n, \lambda$ satisfy the necessary conditions, then there exists an $\operatorname{LHP}(n, n, \lambda)=$ $\left\{\left(Z_{n} \cup \bar{Z}_{n}, \mathcal{A}_{i}\right): 1 \leq i \leq(2 n-1)((n-1) !)^{2} / \lambda\right\}$ by Theorem 5 . For each Hamilton path

$$
C_{j}=\left[x_{0}, \bar{x}_{0}, x_{1}, \bar{x}_{1}, \ldots, x_{n-1}, \bar{x}_{n-1}\right] \in \mathcal{A}_{i},
$$

define two directed Hamilton paths in $K_{n, n}^{*}$ :

$$
\begin{aligned}
C_{j, 1} & =\prec x_{0}, \bar{x}_{0}, x_{1}, \bar{x}_{1}, \ldots, x_{n-1}, \bar{x}_{n-1} \succ \\
C_{j, 2} & =\prec \bar{x}_{n-1}, x_{n-1}, \bar{x}_{n-2}, x_{n-2}, \ldots, \bar{x}_{0}, x_{0} \succ .
\end{aligned}
$$

Let $\mathcal{A}_{i}^{\prime}=\left\{C_{j, 1}, C_{j, 2}: C_{j} \in \mathcal{A}\right\}$, then each $\left\{\left(Z_{n} \cup \bar{Z}_{n}, \mathcal{A}_{i}^{\prime}\right)\right.$ forms a $\operatorname{DHP}(n, n, \lambda)$. Furthermore, it is easy to verify that $\left\{\left(Z_{n} \cup \bar{Z}_{n}, \mathcal{A}_{i}^{\prime}\right): 1 \leq i \leq(2 n-1)((n-1) !)^{2} / \lambda\right\}$ is an $\operatorname{LDHP}(n, n, \lambda)$.

Corollary 3 There exist simple $H P(n, n, \lambda(2 n-1))$ and simple $\operatorname{DHP}(n, n, \lambda(2 n-1))$ if and only if $\leq \lambda \leq((n-1) !)^{2}$.

Acknowledgments The authors would like to thank the referees for their careful reading of the paper and helpful comments.

Open Access This article is distributed under the terms of the Creative Commons Attribution Noncommercial License which permits any noncommercial use, distribution, and reproduction in any medium, provided the original author(s) and source are credited.

\section{References}

1. Bryant, D.: Large sets of Hamilton cycle and path decompositions. Congr. Numer. 135, 147-151 (1998)

2. Kang, Q.: A generalization of Mendelsohn triple systems. Ars Comb. 29C, 207-215 (1990)

3. Kang, Q., Zhao, H.: Large sets of Hamilton cycle decompositions of complete bipartite graphs. Eur. J. Comb. 29, 1492-1501 (2008) 
4. Kang, Q., Chang, Y.: A completion of the spectrum for large sets of transitive triple systems. J. Comb. Theory Ser. A 60, 287-294 (1992)

5. Kang, Q., Lei, J., Chang, Y.: The spectrum of large sets of disjoint Mendelsohn triple systems with any index. J. Comb. Des. 2, 351-358 (1994)

6. Lu, J.: On large sets of disjoint Steiner triple systems I-III. J. Comb. Theory Ser. A 34, 140-182 (1983)

7. Lu, J.: On large sets of disjoint Steiner triple systems IV-VI. J. Comb. Theory Ser. A 37, 136-192 (1984)

8. Teirlinck, L.: A completion of Lu's determination of the spectrum for large sets of disjoint Steiner triple systems. J. Comb. Theory Ser. A 57, 302-305 (1991)

9. Ushio, K., Ohtsubo, Y.: $\hat{C}_{k}$-factorization of symmetric complete bipartite and tripartite multidigraphs. Discrete Math. 223, 393-397 (2000)

10. Zhao, H., Kang, Q.: Large sets of Hamilton cycle and path decompositions. Discret. Math. 308, 4931-4940 (2008)

11. Zhao, H., Kang, Q.: On large sets of almost Hamilton cycle decompositions. J. Comb. Des. 16, 53-69 (2008) 